1 Hacettepe Journal of Mathematics and Statistics

$\bigcap$ Volume 45 (1) (2016), 23-31

\title{
Existence of symmetric positive solutions for a semipositone problem on time scales
}

\author{
S. Gulsan Topal and Arzu Denk*
}

\begin{abstract}
This paper studies the existence of symmetric positive solutions for a second order nonlinear semipositone boundary value problem with integral boundary conditions by applying the Krasnoselskii fixed point theorem. Emphasis is put on the fact that the nonlinear term $f$ may take negative value. An example is presented to demonstrate the application of our main result.
\end{abstract}

Keywords: Positive solution, Symmetric solution, Semipositone problems, Fixed point theorems, Time scales.

2000 AMS Classification: 34B15, 39A10.

Received: 07.03.2013 Accepted: 16.12.2014 Doi : 10.15672/HJMS.20164512479

\section{Introduction}

We will be concerned with proving the existence of at least one symmetric positive solution to the semipositone second order nonlinear boundary value problem on a symmetric time scale $\mathrm{T}$ given by

$$
\begin{aligned}
& {\left[g(t) u^{\triangle}(t)\right]^{\nabla}+\lambda f(t, u(t))=0, \quad t \in(a, b),} \\
& \alpha u(a)-\beta \lim _{t \rightarrow a^{+}} g(t) u^{\triangle}(t)=\int_{a}^{b} h_{1}(s) u(s) \nabla s, \\
& \alpha u(b)+\beta \lim _{t \rightarrow b^{-}} g(t) u^{\triangle}(t)=\int_{a}^{b} h_{2}(s) u(s) \nabla s,
\end{aligned}
$$

where $\lambda>0$ is a parameter, $\alpha, \beta>0, \nabla$-differentiable function $g \in C([a, b],(0, \infty))$ is symmetric on $[a, b], h_{1}, h_{2} \in L^{1}([a, b])$ is nonnegative, symmetric on $[a, b]$ and the continuous function $f:[a, b] \times[0, \infty) \rightarrow R$ satisfies $f(b+a-t, u)=f(t, u)$.

*Department of Mathematics, Ege University, 35100 Bornova, Izmir-Turkey.

Email : f.serap.topal@ege.edu.tr ; arzu.denk@hotmail.com 
A class of boundary value problems with integral boundary conditions arise naturally in thermal condition problems [4], semiconductor problems [7], and hydrodynamic problems [5]. Such problems include two, three and multi-point boundary conditions and have recently been investigated by many authors $[3,6,8,9]$.

The present work is motivated by recent paper [3]. In this paper, Boucherif considered the following second order boundary value problem with integral boundary conditions

$$
\begin{aligned}
& x^{\prime \prime}(t)=f(t, x(t)), \quad 0<t<1, \\
& x(0)-c x^{\prime}(0)=\int_{0}^{1} g_{0}(s) x(s) d s, \\
& x(1)-d x^{\prime}(1)=\int_{0}^{1} g_{1}(s) x(s) d s,
\end{aligned}
$$

where $f:[0,1] \times R \rightarrow R$ is continuous, $g_{0}, g_{1}:[0,1] \rightarrow[0, \infty)$ are continuous and positive, $c$ and $d$ are nonnegative real parameters. The author established some excellent results for the existence of positive solutions to problem (1.4) - (1.6) by using the fixed point theorem in cones.

Throughout this paper $\mathrm{T}$ is a symmetric time scale with $a, b$ are points in $\mathrm{T}$. By an interval $(a, b)$, we always mean the intersection of the real interval $(a, b)$ with the given time scale, that is $(a, b) \cap \mathrm{T}$. Other types of intervals are defined similarly. For the details of basic notions connected to time scales we refer to $[1,2]$.

Now, we present some symmetric definition.

1.1. Definition. A time scale $\mathrm{T}$ is said to be symmetric if for any given $t \in \mathrm{T}$, we have $b+a-t \in \mathrm{T}$.

1.2. Definition. A function $u: \mathrm{T} \rightarrow R$ is said to be symmetric on $\mathrm{T}$ if for any given $t \in \mathrm{T}, u(t)=u(b+a-t)$.

\section{The Preliminary Lemmas}

In this section we collect some preliminary results that will be used in subsequent section.

Throughout the paper we will assume that the following conditions are satisfied:

$\left(H_{1}\right) \quad \alpha, \beta>0$

$\left(H_{2}\right) \quad \nabla$-differentiable function $g \in C([a, b],(0, \infty))$ is symmetric on $[a, b]$,

$\left(H_{3}\right)$ the continuous function $f:[a, b] \times[0, \infty) \rightarrow R$ is semipositone, i.e., $f(t, u)$ needn't be positive for all $(t, u) \in[a, b] \times[0, \infty)$ and $f(., u)$ is symmetric on $[a, b]$ for all $u \geq 0$,

$\left(H_{4}\right) \quad h_{1}, h_{2} \in L^{1}([a, b])$ is nonnegative, symmetric on $[a, b]$ and $A>0$, where $A=$ $\mu+(\beta-K) v_{1}-\beta v_{2}, \quad K=\frac{\mu}{\alpha}, \quad \mu=2 \alpha \beta+\alpha^{2} \int_{a}^{b} \frac{\Delta r}{g(r)}, \quad v_{1}=\int_{a}^{b} h_{1}(\tau) \nabla \tau, \quad v_{2}=$ $\int_{a}^{b} h_{2}(\tau) \nabla \tau$

The lemmas in this section are based on the boundary value problem

$$
-\left[g(t) u^{\triangle}(t)\right]^{\nabla}=p(t), \quad t \in(a, b)
$$

with boundary conditions $(1.2)-(1.3)$.

To prove the main result, we will employ following lemmas.

2.1. Lemma. Let $\left(H_{1}\right),\left(H_{2}\right)$ hold and $A \neq 0$. Then for any $p \in C([a, b])$, the boundary value problem $(2.1)-(1.2)-(1.3)$ has a unique solution u given by 
$u(t)=\int_{a}^{b} H(t, s) p(s) \nabla s$

where

$$
H(t, s)=G(t, s)+B_{1} \int_{a}^{b} G(s, \tau) h_{1}(\tau) \nabla \tau+B_{2} \int_{a}^{b} G(s, \tau) h_{2}(\tau) \nabla \tau
$$

$$
G(t, s)=\frac{1}{\mu} \begin{cases}\left(\beta+\alpha \int_{a}^{s} \frac{\Delta r}{g(r)}\right)\left(\beta+\alpha \int_{t}^{b} \frac{\Delta r}{g(r)}\right), & a \leq s \leq t \leq b, \\ \left(\beta+\alpha \int_{a}^{t} \frac{\Delta r}{g(r)}\right)\left(\beta+\alpha \int_{s}^{b} \frac{\Delta r}{g(r)}\right), & a \leq t \leq s \leq b,\end{cases}
$$

where $\mu=2 \alpha \beta+\alpha^{2} \int_{a}^{b} \frac{\Delta r}{g(r)}, B_{1}=\frac{K-\beta}{A}, B_{2}=\frac{\beta}{A}$.

2.2. Lemma. Assume that $\left(H_{1}\right),\left(H_{2}\right)$ and $\left(H_{4}\right)$ hold. Then we have

(i) $H(t, s)>0, \quad G(t, s)>0$, for $t, s \in[a, b]$,

(ii) $H(b+a-t, b+a-s)=H(t, s), \quad G(b+a-t, b+a-s)=G(t, s)$, for $t, s \in[a, b]$,

(iii) $\frac{1}{\mu} \beta^{2} \gamma \leq H(t, s) \leq H(s, s) \leq \frac{1}{\mu} \gamma D$ and $\frac{1}{\mu} \beta^{2} \leq G(t, s) \leq G(s, s) \leq \frac{1}{\mu} D$, for $t, s \in[a, b]$,

where $D=\left(\beta+\alpha \int_{a}^{b} \frac{\Delta r}{g(r)}\right)^{2}, \gamma=1+B_{1} v_{1}+B_{2} v_{2}$.

Proof. It is clear that $(i)$ hold. Now we prove that $(i i)$ and $(i i i)$ hold. First, we consider (ii). If $t \leq s$, then $b+a-t \geq b+a-s$. Using (2.3) and the assumption $\left(H_{2}\right)$, we get

$$
\begin{aligned}
G(b+a-t, b+a-s)= & \frac{1}{\mu}\left(\beta+\alpha \int_{a}^{b+a-s} \frac{\Delta r}{g(r)}\right)\left(\beta+\alpha \int_{b+a-t}^{b} \frac{\Delta r}{g(r)}\right) \\
& =\frac{1}{\mu}\left(\beta+\alpha \int_{b}^{s} \frac{\Delta(b+a-r)}{g(b+a-r)}\right)\left(\beta+\alpha \int_{t}^{a} \frac{\Delta(b+a-r)}{g(b+a-r)}\right) \\
& =\frac{1}{\mu}\left(\beta+\alpha \int_{s}^{b} \frac{\Delta r}{g(r)}\right)\left(\beta+\alpha \int_{a}^{t} \frac{\Delta r}{g(r)}\right)=G(t, s) .
\end{aligned}
$$

Similarly, we can prove that $G(b+a-t, b+a-s)=G(t, s)$, for $s \leq t$. Thus we have $G(b+a-t, b+a-s)=G(t, s)$, for $t, s \in[a, b]$. Now by $(2.2)$, for $t, s \in[a, b]$, we have

$$
\begin{aligned}
H(b+a-t, b+a-s)= & (b+a-t, b+a-s)+B_{1} \int_{a}^{b} G(b+a-s, \tau) h_{1}(\tau) \nabla \tau \\
& +B_{2} \int_{a}^{b} G(b+a-s, \tau) h_{2}(\tau) \nabla \tau \\
= & G(t, s)+B_{1} \int_{b}^{a} G(b+a-s, b+a-\tau) h_{1}(b+a-\tau) \nabla(b+a-\tau) \\
& +B_{2} \int_{b}^{a} G(b+a-s, b+a-\tau) h_{2}(b+a-\tau) \nabla(b+a-\tau) \\
= & G(t, s)+B_{1} \int_{a}^{b} G(s, \tau) h_{1}(\tau) \nabla \tau+B_{2} \int_{a}^{b} G(s, \tau) h_{2}(\tau) \nabla \tau \\
= & H(t, s) .
\end{aligned}
$$

So $(i i)$ is established. Now we show that ( $i i i)$ holds. In fact, if $t \leq s$, from (2.3) and the assumption $\left(\mathrm{H}_{2}\right)$, then we get 


$$
\begin{aligned}
G(t, s)= & \frac{1}{\mu}\left(\beta+\alpha \int_{a}^{t} \frac{\Delta r}{g(r)}\right)\left(\beta+\alpha \int_{s}^{b} \frac{\Delta r}{g(r)}\right) \leq \frac{1}{\mu}\left(\beta+\alpha \int_{a}^{s} \frac{\Delta r}{g(r)}\right)\left(\beta+\alpha \int_{s}^{b} \frac{\Delta r}{g(r)}\right) \\
& =G(s, s) \\
& \leq \frac{1}{\mu}\left(\beta+\alpha \int_{a}^{b} \frac{\Delta r}{g(r)}\right)\left(\beta+\alpha \int_{a}^{b} \frac{\Delta r}{g(r)}\right)=\frac{1}{\mu}\left(\beta+\alpha \int_{a}^{b} \frac{\Delta r}{g(r)}\right)^{2}=\frac{1}{\mu} D .
\end{aligned}
$$

Similarly, we can prove that $G(t, s) \leq G(s, s) \leq \frac{1}{\mu} D$ for $s \leq t$.

Therefore $G(t, s) \leq G(s, s) \leq \frac{1}{\mu} D$, for $t, s \in[a, b]$. And then, by (2.2), we have

$$
\begin{aligned}
H(t, s) & =G(t, s)+B_{1} \int_{a}^{b} G(s, \tau) h_{1}(\tau) \nabla \tau+B_{2} \int_{a}^{b} G(s, \tau) h_{2}(\tau) \nabla \tau \\
& \leq G(s, s)+B_{1} \int_{a}^{b} G(\tau, \tau) h_{1}(\tau) \nabla \tau+B_{2} \int_{a}^{b} G(\tau, \tau) h_{2}(\tau) \nabla \tau \\
& \leq \frac{1}{\mu} D+\frac{1}{\mu} D B_{1} \int_{a}^{b} h_{1}(\tau) \nabla \tau+\frac{1}{\mu} D B_{2} \int_{a}^{b} h_{2}(\tau) \nabla \tau=\frac{1}{\mu} D\left(1+B_{1} v_{1}+B_{2} v_{2}\right) \\
& =\frac{1}{\mu} D \gamma
\end{aligned}
$$

On the other hand, for $t, s \in[a, b]$, we have

$$
G(t, s) \geq \frac{1}{\mu}\left(\beta+\alpha \int_{a}^{a} \frac{\Delta r}{g(r)}\right)\left(\beta+\alpha \int_{b}^{b} \frac{\Delta r}{g(r)}\right)=\frac{1}{\mu} \beta^{2} .
$$

And then, we get

$$
\begin{aligned}
H(t, s) & =G(t, s)+B_{1} \int_{a}^{b} G(s, \tau) h_{1}(\tau) \nabla \tau+B_{2} \int_{a}^{b} G(s, \tau) h_{2}(\tau) \nabla \tau \\
& \geq \frac{1}{\mu} \beta^{2}+\frac{1}{\mu} \beta^{2} B_{1} \int_{a}^{b} h_{1}(\tau) \nabla \tau+\frac{1}{\mu} \beta^{2} B_{2} \int_{a}^{b} h_{2}(\tau) \nabla \tau=\frac{1}{\mu} \beta^{2} \gamma .
\end{aligned}
$$

Thus for $t, s \in[a, b]$, we have

$$
\frac{1}{\mu} \beta^{2} \gamma \leq H(t, s) \leq H(s, s) \leq \frac{1}{\mu} \gamma D \text { and } \frac{1}{\mu} \beta^{2} \leq G(t, s) \leq G(s, s) \leq \frac{1}{\mu} D .
$$

This completes the proof.

2.3. Lemma. Let $w$ be the unique positive solution of the boundary value problem

$$
\left[g(t) u^{\triangle}(t)\right]^{\nabla}+1=0
$$

with the boundary condition $(1.2)-(1.3)$. Then,

$$
w(t) \leq C \delta, \quad t \in[a, b],
$$

where

$$
\delta=\frac{\beta^{2}}{D}, \quad C=\frac{b-a}{\mu \beta^{2}} D^{2} \gamma
$$

Proof. Using Lemma 2.2, for all $t \in[a, b]$, we have 


$$
w(t)=\int_{a}^{b} H(t, s) \nabla s \leq \frac{1}{\mu} \gamma D \int_{a}^{b} \nabla s=C \delta .
$$

The proof is complete.

Let $E$ denote the Banach space $C[a, b]$ with the norm $\|u\|=\max _{t \in[a, b]}|u(t)|$. Define the cone $P \subset E$ by $P=\{u \in E: u(t)$ is symmetric and $u(t) \geq \delta\|u\|$ for $t \in[a, b]\}$.

To obtain the a positive solution of BVP (1.1) - (1.3), the following fixed point theorem is essential.

2.4. Theorem. Let $E=(E,\|\|$.$) be a Banach space, and let P \subset E$ be a cone in $B$. Assume $\Omega_{1}, \Omega_{2}$ are bounded open subsets of $E$ with $0 \in \Omega_{1}, \overline{\Omega_{1}} \subset \Omega_{2}$, and let$$
S: P \cap\left(\overline{\Omega_{2}} \backslash \Omega_{1}\right) \rightarrow P
$$

be a continuous and completely continuous operator such that, either

(a) $\quad\|S u\| \leq\|u\|, u \in P \cap \partial \Omega_{1}$, and $\|S u\| \geq\|u\|, u \in P \cap \partial \Omega_{2}$, or

(b) $\quad\|S u\| \geq\|u\|, u \in P \cap \partial \Omega_{1}$, and $\|S u\| \leq\|u\|, u \in P \cap \partial \Omega_{2}$.

Then $S$ has a fixed point in $P \cap\left(\overline{\Omega_{2}} \backslash \Omega_{1}\right)$.

\section{Main Results}

In this section, we apply the Krasnoselskii fixed point theorem to obtain the existence of at least one symmetric positive solution for the nonlinear boundary value problem $(1.1)-(1.3)$.

The main result of this paper is following:

3.1. Theorem. Let $\left(H_{1}\right)-\left(H_{4}\right)$ hold. Assume that

$\left(C_{1}\right)$ There exists a constant $M>0$ such that $f(t, u) \geq-M$ for all $(t, u) \in[a, b] \times$ $[0, \infty)$

$\left(C_{2}\right)$ There exist $\lim _{u \rightarrow \infty} \frac{\mathbb{f}_{1}(t, t a) \in(a, b)}{u}=$ such that

uniformly on $\left[t_{1}, t_{2}\right]$,

$\left(C_{3}\right) r$ is a given positive real number and the parameter $\lambda$ satisfies

$$
0<\lambda \leq \eta:=\min \left\{\frac{r}{M_{1}\|w\|}, \frac{r}{2 M C}\right\}
$$

where $M_{1}=\max \{f(t, u)+M:(t, u) \in[a, b] \times[0, r]\}$.

Then the boundary value problem (1.1) - (1.3) has at least one symmetric positive solution $u$ such that $\|u\| \geq \frac{r}{2}$.

Proof. Let $x(t)=\lambda M w(t)$, where $w$ is the unique solution of the boundary value problem $(2.4)-(1.2)-(1.3)$.

We shall show that the following boundary value problem

$$
\begin{gathered}
{\left[g(t) y^{\triangle}(t)\right]^{\nabla}+\lambda F(t, y(t)-x(t))=0, t \in(a, b),} \\
\alpha y(a)-\beta \lim _{t \rightarrow a^{+}} g(t) y^{\triangle}(t)=\int_{a}^{b} h_{1}(s) y(s) \nabla s, \\
\alpha y(b)+\beta \lim _{t \rightarrow b^{-}} g(t) y^{\triangle}(t)=\int_{a}^{b} h_{2}(s) y(s) \nabla s,
\end{gathered}
$$

where 


$$
F(t, z)= \begin{cases}f(t, z)+M, & z \geq 0 \\ f(t, 0)+M, & z \leq 0\end{cases}
$$

has at least one positive solution. Thereafter we shall obtain at least one positive solution for the boundary value problem (1.1) - (1.3).

It is well known that the existence of positive solution to the boundary value problem $(3.2)-(3.4)$ is equivalent to the existence of fixed point of the operator $S$. So we shall seek a fixed point of $S$ in our cone $P$ where the operator $S: E \rightarrow E$ is defined by

$$
S y(t)=\lambda \int_{a}^{b} H(t, s) F(s, y(s)-x(s)) \nabla s, \quad t \in[a, b] .
$$

First, it is obvious that $S$ is continuous and completely continuous.

Now we shall prove that $S(P) \subseteq P$. Let $y \in P$. Then, using Lemma 2.2 , we get for $t \in[a, b]$,

$$
S y(t)=\lambda \int_{a}^{b} H(t, s) F(s, y(s)-x(s)) \nabla s \leq \frac{\lambda}{\mu} \gamma D \int_{a}^{b} F(s, y(s)-x(s)) \nabla s,
$$

and so

$$
\|S y\| \leq \frac{\lambda}{\mu} \gamma D \int_{a}^{b} F(s, y(s)-x(s)) \nabla s
$$

Now, using Lemma 2.2 and (3.5), we obtain for $t \in[a, b]$,

$$
\begin{aligned}
S y(t) & =\lambda \int_{a}^{b} H(t, s) F(s, y(s)-x(s)) \nabla s \geq \frac{\lambda}{\mu} \beta^{2} \gamma \int_{a}^{b} F(s, y(s)-x(s)) \nabla s \\
& =\frac{\lambda}{\mu} \delta \gamma D \int_{a}^{b} F(s, y(s)-x(s)) \nabla s \geq \delta\|S y\| .
\end{aligned}
$$

On the other hand, noticing $y(t), x(t)$ and $f(t, u)$ are symmetric on $[a, b]$, we have

$$
\begin{aligned}
S y(b+a-t) & =\lambda \int_{a}^{b} H(b+a-t, s) F(s, y(s)-x(s)) \nabla s \\
& =\lambda \int_{a}^{b} H(b+a-t, s)(f(s, y(s)-x(s))+M) \nabla s \\
& =\lambda \int_{b}^{a} H(b+a-t, b+a-s)(f(s,(y-x)(b+a-s))+M) \nabla(b+a-s) \\
& =\lambda \int_{a}^{b} H(t, s)(f(s,(y-x)(s))+M) \nabla s \\
& =\lambda \int_{a}^{b} H(t, s) F(s,(y-x)(s)) \nabla s=S y(t)
\end{aligned}
$$

Therefore $S y$ is symmetric.

So, we get $S(P) \subseteq P$.

Let $\Omega_{1}=\{y \in E:\|y\|<r\}$. We shall prove that $\|S y\| \leq\|y\|$ for $y \in P \cap \partial \Omega_{1}$. If $y \in P \cap \partial \Omega_{1}$, then $\|y\|=r$. By definition and (3.1), we find for $t \in[a, b]$,

$$
S y(t)=\lambda \int_{a}^{b} H(t, s) F(s, y(s)-x(s)) \nabla s \leq \lambda M_{1} \int_{a}^{b} H(t, s) \nabla s \leq \lambda M_{1}\|w\| \leq r .
$$


Therefore, we get $\|S y\| \leq r=\|y\|$ for $y \in P \cap \partial \Omega_{1}$.

Let $K$ be a positive real number such that

$$
\frac{1}{2} \lambda K\left(t_{2}-t_{1}\right) \delta \frac{1}{\mu} \beta^{2} \gamma>1
$$

In view of $\left(C_{2}\right)$, there exists $N>0$ such that for all $z \geq N$ and $t \in\left[t_{1}, t_{2}\right]$,

$$
F(t, z)=f(t, z)+M \geq K z
$$

Now, set

$$
R=r+\frac{2 N}{\delta} .
$$

Let $\Omega_{2}=\{y \in E:\|y\|<R\}$. We shall prove that $\|S y\| \geq\|y\|$ for $y \in P \cap \partial \Omega_{2}$. If $y \in P \cap \partial \Omega_{2}$, then $\|y\|=R$. So from Lemma 2.3 and the fact that $y \in P$, we get for $t \in[a, b]$,

$$
x(t)=\lambda M w(t) \leq \lambda M C \delta \leq \lambda M C \frac{y(t)}{R} .
$$

This implies for $t \in[a, b]$,

$$
y(t)-x(t) \geq\left(1-\frac{\lambda M C}{R}\right) y(t) \geq\left(1-\frac{\lambda M C}{R}\right) \delta R,
$$

and, from (3.1) and (3.8), we get for $t \in\left[t_{1}, t_{2}\right]$,

$$
y(t)-x(t) \geq \frac{1}{2} R \delta \geq N
$$

Thus, by (3.7) and (3.9), we see that for $t \in\left[t_{1}, t_{2}\right]$,

$$
F(t, y(t)-x(t)) \geq K(y(t)-x(t)) \geq \frac{1}{2} K R \delta .
$$

Considering Lemma 2.2 and (3.10), we get for $t \in[a, b]$,

$$
\begin{aligned}
S y(t) & =\lambda \int_{a}^{b} H(t, s) F(s, y(s)-x(s)) \nabla s \geq \lambda \frac{1}{\mu} \beta^{2} \gamma \int_{t_{1}}^{t_{2}} F(s, y(s)-x(s)) \nabla s \\
& \geq \frac{1}{2 \mu} \lambda K R \delta \beta^{2} \gamma \int_{t_{1}}^{t_{2}} \nabla s
\end{aligned}
$$

and so by (3.6),

$$
\|S y\| \geq \frac{1}{2 \mu} \lambda K R\left(t_{2}-t_{1}\right) \delta \beta^{2} \gamma \geq R .
$$

Therefore, we get $\|S y\| \geq R=\|y\|$ for $y \in P \cap \partial \Omega_{2}$.

Then it follows from Theorem 2.1 that $\mathrm{S}$ has a fixed point $y \in P$ such that

$$
r \leq\|y\| \leq R
$$

Moreover, using (3.1), (3.11) and Lemma 2.3, we obtain for $t \in[a, b]$, 


$$
y(t) \geq \delta\|y\| \geq r \delta \geq 2 \lambda M C \delta \geq 2 \lambda M w(t)=2 x(t)
$$

Hence,

$$
u(t)=y(t)-x(t) \geq 0, \quad t \in[a, b] .
$$

On the other hand, $u(t)$ is symmetric on $[a, b]$ since $y$ and $x$ are symmetric.

Now, we shall prove that $u$ is a positive solution of the boundary value problem (1.1) (1.3). Since $y$ is a fixed point of the operator $S$,

or

$$
S y(t)=y(t), \quad t \in[a, b],
$$

$$
\begin{aligned}
y(t) & =S y(t)=\lambda \int_{a}^{b} H(t, s) F(s, y(s)-x(s)) \nabla s \\
& =\lambda \int_{a}^{b} H(t, s)(f(s, y(s)-x(s))+M) \nabla s
\end{aligned}
$$

Noticing that,

$$
w(t)=\int_{a}^{b} H(t, s) \nabla s
$$

we have for $t \in[a, b]$,

$$
y(t)=\lambda \int_{a}^{b} H(t, s) f(s, y(s)-x(s)) \nabla s+\lambda M w(t),
$$

or

$$
y(t)-x(t)=\lambda \int_{a}^{b} H(t, s) f(s, y(s)-x(s)) \nabla s,
$$

and hence

$$
u(t)=\lambda \int_{a}^{b} H(t, s) f(s, u(s)) \nabla s .
$$

This shows that $u$ is a symmetric positive solution of the boundary value problem of (1.1) - (1.3). In addition, from (3.11) and (3.12), it follows that

$$
\|u\| \geq \frac{\|y\|}{2} \geq \frac{r}{2}
$$

3.2. Example. Let $T=Z$. Consider the following boundary value problem

$$
\begin{gathered}
{\left[\frac{100}{t^{2}+1} u^{\triangle}(t)\right]^{\nabla}+\lambda\left(b e^{u} \cos ^{2} t-t^{2}\right)=0, t \in(-3,3),} \\
25 u(-3)-5 \lim _{t \rightarrow-3^{+}} \frac{100}{t^{2}+1} u^{\triangle}(t)=\int_{-3}^{3} u(s) \cosh s \nabla s, \\
25 u(3)+5 \lim _{t \rightarrow 3^{-}} \frac{100}{t^{2}+1} u^{\triangle}(t)=\int_{-3}^{3} u(s) \cosh s \nabla s,
\end{gathered}
$$

where $b>0, \alpha=25, \beta=5, h_{1}(t)=h_{2}(t)=\cosh t, g(t)=\frac{100}{t^{2}+1}, f(t, u(t))=b e^{u} \cos ^{2} t-$ $t^{2}$. It is obvious that $f$ satisfies the conditions $\left(C_{2}\right)$ and $\left(H_{3}\right)$.

Now we shall obtain the constants $M$ and $M_{1}$. Clearly, for all $(t, u) \in[-3,3] \times[0, \infty)$, we get

$$
f(t, u)=b e^{u} \cos ^{2} t-t^{2} \geq-t^{2} \geq-9 \text { and so we can choose the constant } M=9 .
$$




$$
M_{1}=\max _{(t, u) \in[-3,3] \times[0, r]} b e^{u} \cos ^{2} t-t^{2}+M=b e^{r}+M .
$$

It follows from a direct calculation that

$$
\begin{aligned}
& v_{1}=v_{2}=\int_{-3}^{3} h_{1}(s) \nabla s \cong 21.5, \mu=2 \alpha \beta+\alpha^{2} \int_{-3}^{3} \frac{\Delta r}{g(r)} \cong 406.2 \\
& D=\left(\beta+\alpha \int_{-3}^{3} \frac{\Delta r}{g(r)}\right)^{2} \cong 126.6, A=\mu+(\beta-K) v_{1}-\beta v_{2} \cong 56,87 \\
& B_{1}=\frac{K-\beta}{A} \cong 0.198, B_{2}=\frac{\beta}{A} \cong 0.088, \gamma=1+B_{1} v_{1}+B_{2} v_{2} \cong 7.15 \\
& C=\frac{6}{\mu \beta^{2}} D^{2} \gamma \cong 67.71
\end{aligned}
$$

Then by Theorem 3.1, we see that the boundary value problem $(3.13)-(3.15)$ has at least one symmetric positive solution $u$ such that $\|u\| \geq \frac{r}{2}$ for any $\lambda \in(0, \eta]$ where $\eta:=\min \left\{\frac{r}{M_{1}\|w\|}, \frac{r}{2 M C}\right\}, r$ is a given positive number and $w$ is the unique positive solution of the boundary value problem $\left[\frac{100}{t^{2}+1} u^{\triangle}(t)\right]^{\nabla}+1=0$ with the boundary condition $(3.14)-(3.15)$.

\section{References}

[1] M. Bohner, A. Peterson, Dynamic Equations on Time Scales: An Introduction with Applications, Birkhauser, Boston, Cambridge, MA (2001).

[2] M. Bohner, A. Peterson, Advances in Dynamic Equations on Time Scales, Birkhauser, Boston, Cambridge, MA (2003).

[3] A. Boucherif, Second-order boundary value problems with integral boundary conditions, Nonlinear Anal. 70 (2009) 364-371.

[4] J. R. Cannon, The solution of the heat equation subject to the specification of energy, Quart. Appl. Math. 21 (2) (1963) 155-160.

[5] R. Yu. Chegis, Numerical solution of a heat conduction problem with an integral boundary condition, Litovsk. Math. Sb. 24 (1984) 209-215.

[6] J. Henderson, H. B. Thompson, Multiple symmetric positive solutions for a second order boundary value problem, Proc. Amer. Math. Soc. 128 (2000) 2373-2379.

[7] N. I. Ionkin, Solution of a boundary value problem in heat conduction theory with nonlocal boundary conditions, Differential Equations 13 (1977) 294-304.

[8] F. Li, Y. Zhang, Multiple symmetric nonnegative solutions of second-order ordinary differential equations, Appl. Math. Lett. 17 (2004) 261-267.

[9] Y. Sun, Optimal existence criteria for symmetric positive solutions to a three-point boundary value problem, Nonlinear Anal. 66 (2007) 1051-1063. 
